\title{
A Nested Game-Based Optimization Framework for Electricity Retailers in the Smart Grid with Residential Users and PEVs
}

\author{
Yang Li, Yanzhi Wang, Shahin Nazarian, and Massoud Pedram \\ University of Southern California \\ Los Angeles, CA USA \\ \{yli760, yanzhiwa, shahin, pedram $\} @$ usc.edu
}

\begin{abstract}
In the smart grid, real-time pricing policy is an important mechanism for incentivizing the consumers to dynamically change or shift their electricity consumption, thereby improving the reliability of the grid. Retailers are incorporated to the smart grid with distributed control mechanism in order to reduce the amount of communication overhead associated with the direction interaction between utility companies and consumers. The retailer procures electricity from both traditional and renewable energy sources, and sells it to its consumers. The consumers include residential users that can only consume power, and plug-in electric vehicles (PEVs) that can either consume power or supply power stored in its battery to the grid. In this work, a novel four-stage nested game model is proposed to model the interaction of the electricity retailer, utility companies, and consumers. The objective of the retailer is to maximize its overall profit as well as perform frequency regulation, whereas the goal of each consumer is to maximize a predefined utility function. In the game theoretic framework, the retailer should decide the amounts of electricity purchased from the renewable and traditional energy sources, respectively, as well as the real-time pricing scheme for its consumers. The consumers will react to the pricing mechanism and maximize their utility functions by adjusting the electricity demand. The optimal solution of the nested game is provided through: (i) finding the subgame perfect equilibrium (SPE) of all the consumers, and (ii) optimizing the retailer's action using the backward induction method. Experimental results demonstrate the effectiveness of the proposed game theoretic modeling and optimization framework.
\end{abstract}

\section{INTRODUCTION}

With the increasing demand of energy worldwide, the design of future electricity systems has become a big concern [1]. Traditional power grids are usually utilized to deliver electricity from central generators to a large number of users [2]. In contrast, the recently proposed smart grid uses two-way flows of electricity and information to create an automated and distributed energy delivery network [2]. Smart grid takes advantage of the modern communication system to gather information from consumers and suppliers in order to improve efficiency, reliability, and sustainability of the power grid. This outstanding merit makes smart grid an ideal choice to gradually replace the energy inefficient traditional power grid. The improvement of energy efficiency in electricity infrastructure based on smart grid will alleviate the problem of energy deficiency faced by many countries all over the world [1].

Currently, there are many researches working on the modeling and application of smart grid in both industry and academia. Most of them focus on the management of smart grid and demand side optimization to enhance efficiency and reliability. Reference work [3], [4] proposed a centralized control optimization method to achieve the optimal energy efficiency. It provides important insights on how to efficiently match the energy supply of power grid and demand of consumers during peak and off-peak hours without wasting the generated energy. However, it may be difficult to realize the assumptions behind the centralized control method: (i) the smart grid central controller can directly control the energy usage of each consumer, and (ii) each consumer needs to provide their demand information to the energy provider in advance, which is especially difficult for the consumers with high mobility such as plug-in electric vehicles (PEVs). Therefore, a decentralized smart grid control framework is a proper candidate to overcome those shortcomings [5].

Decentralized/distributed control mechanism in the smart grid requires the utility companies to employ day-ahead pricing or real-time pricing schemes in order to incentivize the consumers to perform demand side management (DSM), e.g., shifting their loads from peak hours to off-peak hours. Both utility companies and energy users aim at maximizing their own profits or minimizing costs [5]. When day-ahead pricing is applied, the utility company announces the time-of-use dependent price signal over the next billing period (the next day), and the customers respond to the price signal by adjusting their load demand over the whole billing period [5]. When realtime pricing is applied, the utility company announces the relationship (usually a superlinear function) between the electricity price and the total load demand over the next time slot (typically a few minutes to one hour.) This pricing scheme fits very well for applications such as vehicle-to-grid (V2G) systems (e.g., [6]-[8]). Since the electricity price is dependent on the total load demand of all the users, game theory has been proposed as the analysis tool for the distributed users in the real-time pricing scenario [6], [11]. Consumers are usually modeled as rational players in a non-cooperative game and their actions are the load demand values. The Nash equilibrium among all the consumers is derived from the relationship between electricity price and total load demand.

Retailers are incorporated to the smart grid with distributed control mechanism in order to reduce the amount of computation and communication overheads associated with the direct interaction between utility companies and consumers. A retailer is an intermediary between the utility companies and consumers. In an electricity market, retailers procure electricity 
from various energy sources, including both renewable and non-renewable ones, and then sell the electricity to its customers [12]. Due to the intermittent nature of the electricity generated from renewable power sources and the fluctuation of power demand, the retailer needs to judiciously determine the amount of electricity they procure from different sources, in order to (i) increase the reliability of power supply, (ii) reduce the cost from purchasing electricity, and (iii) provide frequency regulation for the grid [6]. A Stackelberg game is adopted in [12] to model the interactions between the electricity retailer and consumers. The backward induction method, which is a standard solution method for Stackelberg games [13], is applied to derive the optimal amount of electricity purchased and the electricity price set by the retailer.

In this paper, we consider an extension over [12]. The proposed system model is comprised of both renewable and non-renewable (traditional) energy sources, a retailer, and a set of energy consumers. The retailer buys electricity from both energy sources and applies real-time pricing for the consumers. The consumers can be divided into two categories: residential users that can only consume power and PEVs that can either consume power or supply power stored in its battery to the grid. We present a four-stage nested game model for the interaction of the electricity retailer, utility companies, and consumers. The objective of the retailer is to maximize its overall profit as well as perform frequency regulation. Here the PEVs' battery can serve as regulation unit through charging and discharging process which is auxiliary to the original frequency regulation mechanism built inside retailer's power grid. If the number of PEVs is relatively large, retailer will benefit from this in the sense that it can avoid using high cost frequency regulation facilities such as turbine speed governors. Meanwhile, the goal of each consumer is to maximize a predefined utility function. The retailer is involved in the first three steps of decision making in the nested game. In Step I and II, the retailer decides the amounts of electricity purchased from the renewable power source and the traditional power source, respectively. In Step III, the retailer provides the optimal real-time pricing scheme to its consumers to specify the relationship between energy price and total load demand, based on all the previous decisions. In the last stage (Stage IV), all the consumers will react to the pricing mechanism and maximize their utility functions by adjusting the electricity demand. We propose the optimal solution of the nested game for both the retailer and the consumers. First, we find the subgame perfect equilibrium (SPE) for all the consumers (i.e., residential users and PEVs) in Stage IV. Then we optimize the retailer's action in the first three stages using the backward induction method. We use the dynamic programming method to reduce the computation and storage complexity during backward induction.

The rest of this paper is organized as follows. The proposed system model is described in Section II. The nested game formulation and its analysis are discussed in Section III. Section IV provides the backward induction-based optimization procedure of the nested game. Simulation results are presented in Section V, and we conclude in Section VI.

\section{SYSTEM MODEL}

In this section, we present the system model. Consider a smart grid system with both renewable and non-renewable utility companies, various consumers, and an electricity retailer. The retailer plays an important role in the system. It acquires electricity from the utility companies at certain price levels and supplies its customers (consumers). The retailer provides the real-time pricing signal to specify the relationship between the electricity price and the total load demand over the next time slot. In reality, a two-way communication infrastructure built in the smart grid can help the retailer to announce such real-time information to the consumers. There are two categories of consumers in the smart grid system model. One kind is the residential users that can only consume power. The other kind is PEVs that have a built-in battery as the energy storage. They can not only buy electricity from the retailer to charge the battery but also sell energy to the retailer to get certain amount of profit when they have excessive stored energy. All the consumers will react to the pricing signal and adjust their power demands from the grid.

\section{A. Energy Sources (Utility Companies)}

In our model, the retailer has two main sources to purchase electricity through power grid. One is from the renewable power sources such as wind or solar power generation facilities. The other is from the traditional power sources such as coal-fired power stations. From the perspective of cost per unit electricity, the renewable power source is a good choice for the retailer to lower down the cost of purchasing electricity. However, in terms of the reliability of power supply, the traditional power source is better. The reason is that the renewable power (e.g., wind or solar) generations are difficult to maintain a high quaility of continuous and steady power supply since they largely depend on the weather condition and other factors. This creates a reliabity issue when the retailer tries to buy electricity from such energy sources.

In the proposed model, let $P_{\mathbf{R}}$ denote the reliability factor of the renewable energy source, i.e., the probability that the renewable power supply purchased by the retailer turns out to be reliable, where the subscript $\mathbf{R}$ represents for "renewable". Let $D_{0, b u y}^{\mathbf{R}}$ and $D_{0, a c t u a l}^{\mathbf{R}}$ denote the amounts of renewable energy purchased and actually received, respectively, by the retailer. $D_{0, \text { actual }}^{\mathbf{R}}$ is a random variable, and we have the following relationship:

$$
D_{0, a c t u a l}^{\mathbf{R}}=\left\{\begin{array}{lr}
D_{0, b u y}^{\mathbf{R}} & \text { with probability } P_{\mathbf{R}} \\
0 & \text { with probability } 1-P_{\mathbf{R}}
\end{array}\right.
$$

On the other hand, the amount of purchased (and received) energy from the traditional energy source is denoted by $D_{0, \text { actual }}^{\mathrm{NR}}$, where the subscript $\mathbf{N R}$ represents for "nonrenewable". Then the total amount of energy $D_{0}$ received by the retailer from the power sources is given by:

$$
D_{0}=D_{0, \text { actual }}^{\mathbf{R}}+D_{0, \text { actual }}^{\mathrm{NR}}
$$

Moreover, let price ${ }^{\mathbf{R}}$ and price ${ }^{\mathbf{N R}}$ denote the unit energy prices from the renewable and traditional power sources, respectively, and obviously price $e^{\mathbf{R}}<$ price $^{\mathbf{N R}}$. The total energy cost that the retailer pays for purchasing energy is: 


$$
\text { price }^{\mathbf{R}} \cdot D_{0, b u y}^{\mathbf{R}}+\text { price }^{\mathbf{N R}} \cdot D_{0, a c t u a l}^{\mathbf{N R}}
$$

A desirable plan for the retailer is to purchase a portion of electricity from renewable resources and the rest from traditional power plants. With a proper tradeoff of energy cost and reliability, the retailer could enhance its overall profit.

\section{B. Consumer Model and Real-Time Pricing}

Let $N$ denote the number of consumers associated with the retailer. Let $d_{i}$ denote the energy demand of user $i$ in the time slot of interest. If $d_{i} \geq 0$, the $i^{\text {th }}$ user is consuming energy purchased from the retailer. If $d_{i}<0$, the $i^{\text {th }}$ user is selling energy to the retailer and this negative energy demand only applies to PEVs. Let $D$ represents the total demand of all the users, and then we have:

$$
D=\sum_{i=1}^{N} d_{i}
$$

Furthermore, let $D_{\text {neg }}$ represent the sum of all the negative energy demands, and we have:

$$
D_{\text {neg }}=\sum_{i=1}^{N} d_{i} \mathbf{I}\left[d_{i}<0\right]
$$

where $\mathbf{I}[x]$ is an indicator function that equals to 1 if the Boolean variable $x$ is true, and equals to 0 otherwise.

The retailer serves a group of consumers and determines the pricing policy for the consumers to purchase or sell electricity. It should balance the energy demand (from the energy sources) and supply (to the consumers) in order to perform proper frequency regulation [6]. Balancing demand and supply is a difficult task for the retailer because of the fluctuation in load demands, especially for PEVs with high mobility. The real-time pricing policy is adopted by the retailer to achieve this goal. The basic principle is that if there is an increase or decrease in the overall energy consumption with respect to the total amount of energy procured by the retailer, it will adjust the electricity prices to encourage users to use less or more electricity, respectively.

Given these reasons and principles, the real-time pricing policy is described as follows. Let $p_{c}$ and $p_{d}$ denote the unit energy prices for the consumer to purchase energy from or sell energy to the retailer, respectively. Then the relationship between the prices $p_{c}, p_{d}$ and the total load demand $D$ can be mathematically described as follows:

$$
\begin{aligned}
& p_{c}(D)=\beta\left(D-D_{0}\right)+p_{B} \\
& p_{d}(D)=\gamma\left(D-D_{0}\right)+p_{B}
\end{aligned}
$$

where $\beta$ and $\gamma$ are coefficients determined by the retailer. $p_{B}$ represents the base price for the consumer to purchase and sell electricity when $D=D_{0}$. As we can see from (6) and (7), if the total load demand $D$ is higher than $D_{0}, p_{c}$ will be increased to discourage the users from consuming more energy. Moreover, $p_{d}$ will also be increased to attract more users (PEVs) to discharge their batteries and contribute to the energy supply of retailer. On the other hand, if the current total demand $D$ is less than $D_{0}$, a preferable pricing scheme is to encourage consumers to buy more electricity and sell less. Therefore, the price of buying electricity $p_{c}$ will be lower and the benefit of selling electricity $p_{d}$ will also be less.

At the demand side, each residential user cares about two aspects. One is to minimize the cost when the user purchases electricity from the retailer. The other is to maximize its own satisfaction level. As a combination of these two effects, each $i^{\text {th }}$ user (if it is a residential user) maximizes a utility function with the following form:

$$
U R_{i}\left(d_{i}, p_{c}\right)=-a_{i} d_{i}^{2}+e_{i} d_{i}-p_{c}\left(d_{i}, d_{-i}\right) \cdot d_{i}
$$

where $d_{-i}$ denotes the energy demand profile of the other users than user $i$. We know that $p_{c}\left(d_{i}, d_{-i}\right)$ is a function of both $d_{i}$ and $d_{-i}$ because it is an increasing function of the total demand $D$ as shown in (6). Moreover, $a_{i}$ and $e_{i}$ in (8) are positive coefficients. $a_{i}$ and $e_{i}$ may be different for different residential users. This type of utility function is a concave function and has a most desirable $d_{i}$ value to get the maximum payoff when $d_{-i}$ is given. The most desirable value of $d_{i}$ is determined by $a_{i}, e_{i}$ and $p_{c}\left(d_{i}, d_{-i}\right)$. Because residential users can only buy electricity from the retailer, $d_{i} \geq 0$ is a constraint during the utility optimization process of residential users.

For the PEVs with a battery storage bank, they can buy electricity from the retailer at price $p_{c}$ as well as sell its electricity to the retailer at price $p_{d}$ to gain some profit. We use the following type of utility functions for the PEVs:

$$
U_{E V}\left(d_{i}, p\right)=\varepsilon_{i} \sqrt{S_{i n i, i}+d_{i}}-p \cdot d_{i}
$$

where

$$
p= \begin{cases}p_{c}\left(d_{i}, d_{-i}\right) & \left(d_{i} \geq 0\right) \\ p_{d}\left(d_{i}, d_{-i}\right) & \left(d_{i}<0\right)\end{cases}
$$

where $\varepsilon_{i}\left(\varepsilon_{i}>0\right)$ is a coefficient for the utility function of the $i^{\text {th }}$ user (if it is a PEV.) $S_{i n i, i}$ is the amount of energy initially stored in the PEV's battery. Clearly, there are different initial energy levels among all the PEVs. The PEVs want to maintain enough charge in the battery and sell the excessive amount of electricity to the retailer in order to gain some profit. The first term of (9) shows that each PEV intends to maximize its final energy level stored in the battery after charging/discharging, whereas the second term of (9) shows that each PEV also wants to minimize the electricity cost for charging or maximize the revenue from discharging the battery. Each PEV needs to determine whether to buy or sell electricity based on the realtime pricing policy, the initial energy level, and also other users, in order to maximize its own utility.

\section{Retailer's Utility Function}

For the retailer, the goal is to maximize its overall profit as well as perform frequency regulation through matching the power supply and demand. Therefore, the retailer needs to minimize the cost of buying electricity from power grid and the mismatch between $D$ and $D_{0}$, as well as maximize the revenue of selling electricity to its users. According to the above objectives, we formulate its utility (payoff) function as follows: 
$U_{\text {retailer }}$

$=\left(D-D_{n e g}\right) \cdot p_{c}(D)+D_{n e g} \cdot p_{d}(D)$

$-D_{0, \text { actual }}^{\mathbf{N R}} \cdot$ price $^{\mathbf{N R}}-D_{0, \text { buy }}^{\mathbf{R}} \cdot$ price $^{\mathbf{R}}-\theta \cdot\left(D-D_{0}\right)^{2}$

In (11), $\left(D-D_{n e g}\right) \cdot p_{c}(D)+D_{n e g} \cdot p_{d}(D)$ is the sum of the retailer's revenue from selling electricity to the users (and purchasing electricity from some users.) $D_{0, \text { actual }}^{\mathbf{N R}} \cdot$ price $^{\mathbf{N R}}$ and $D_{0, b u y}^{\mathbf{R}} \cdot$ price $^{\mathbf{R}}$ represent the cost of purchasing electricity from traditional power sources and renewable power sources, respectively. $\left|D-D_{0}\right|$ is the mismatch between total energy demand from all users and the total amount of electricity energy purchased by the retailer. In (11), $\theta \cdot\left(D-D_{0}\right)^{2}$ is used in the formulation where $\theta(\theta>0)$ is a constant, and the retailer will get a higher payoff by minimizing this term.

\section{NESTED GAME FORMULATION AND ANALYSIS}

Based on the system model, we present a four-stage nested game based formulation to model the hierarchical decision making process and the interactions between the retailer and various users. The proposed nested game formulation is illustrated in Fig. 1.

\begin{tabular}{|c|c|}
\hline Stage I: & Retailer determines $D_{0, b y}^{R}$ \\
\hline Stage II: & $\begin{array}{l}\text { Retailer determines non- } \\
\text { renewable energy }\end{array}$ \\
\hline & $\downarrow$ \\
\hline Stage III: & $\begin{array}{l}\text { Retailer determines the } \\
\text { factor } \beta \text { and } \gamma\end{array}$ \\
\hline & $\downarrow$ \\
\hline Stage IV: & Users determines demand $d_{i}$ \\
\hline
\end{tabular}

Fig. 1. Four-stage nested game based formulation for the interaction system.

As shown in Fig. 1, we provide an overview of the fourstage nested game as follows:

- Stage I: The retailer determines the amount of electricity to purchase from renewable energy sources, i.e. $D_{0, b u y}^{\mathbf{R}}$, based on the reliability factor $P_{\mathbf{R}}$.

- Stage II: The retailer determines the amount of electricity to purchase from traditional energy sources, $D_{0, \text { actual }}^{\mathbf{N R}}$, based on the amount of electricity $D_{0, \text { actual }}^{\mathbf{R}}$ acquired from the renewable sources in Stage I. The total amount of purchased electricity $D_{0}$ is also decided in this stage since $D_{0}=D_{0, \text { actual }}^{\mathbf{R}}+D_{0, \text { actual }}^{\mathbf{N R}}$.

- $\quad$ Stage III: The retailer provides a pricing mechanism by determining the factors $\beta$ and $\gamma$ based on $D_{0}$. The goal in this stage is to maximize the retailer's profit as well as minimize the mismatch between $D_{0}$ and $D$.

- Stage IV: Based on the pricing mechanism offered by the retailer in Stage III, each user $i$ (residential user or
PEV) will maxmize its own utility function by determining its demand $d_{i}$ with awareness of the pricing mechanism and the other users. This forms a non-cooperative subgame of the nested game. We prove the existence and uniqueness of Nash equilibrium in this game, which is the SPE of the overall nested game.

Backward induction [13] encapsulates the sequential rationality of decision making and is used as a powerful technique to obtain the best strategies for the players in each stage of the nested game (or a sequential game in general.) It reflects the sequential dependencies of decisions in each stage of the nested game. Based on the backward induction principle, we first find the SPE in Stage IV among all the residential users and PEVs given the retailer's pricing mechanism. Then we move backward to analyze and optimize the retailer's strategies in the first three stages. We will discuss the optimization procedure in detail as follow.

\section{A. Game Theoretic Optimization in Stage IV}

In this stage, each user will adjust its demand $d_{i}$ according to the $\beta$ and $\gamma$ values (provided by the retailer in Stage III) and the awareness of other users. As a self-interest individual, each $i^{\text {th }}$ user wants to maximize its own utility by finding a proper value $d_{i}$. The total demand $D$, which depends on the energy demands of all users, will also affect $p_{c}$ and $p_{d}$ through (6) and (7). Hence, the interaction of users forms a normal-form game where all users take action simultaneously. We name this game the Energy Demand Optimization (EDO) game. The EDO game is a subgame of the overall four-stage nested game.

The Nash equilibrium of a normal-form game is the optimal strategy profile for all the players in the sense that no player can find a better strategy (i.e., the value of $d_{i}$ ) if he deviates from the current strategy unilaterally. In other words, no player (residential user or PEV) will have incentive to leave this strategy in the Nash equilibrium. Hence, Nash equilibrium is of particular interest to a non-cooperative normal-form game. The Nash equilibrium of the EDO game is the SPE of the four-stage nested game. Next, we prove the existence and uniqueness of the Nash equilibrium in the EDO game if $\beta=\gamma$, i.e., the prices $p_{c}(D)$ and $p_{d}(D)$ are equal to each other. The unique Nash equilibrium can be found using standard convex optimization technique [15], as described in Algorithm 1. On the other hand, Algorithm 1 will find the approximate best response for each user in the more general case of $\beta \neq \gamma$.

Theorem I: The Nash equilibrium of the EDO game exists and is unique if $\beta=\gamma$.

Proof: According to the utility function of each consumer in (8) and (9), we are essentially trying to maximize a strictly concave utility function for each player on a closed convex set. Therefore, from the first and third theorem in [14], the existence and uniqueness of the Nash equilibrium is proved. 


\section{Algorithm 1: Find the SPE solution for the subgame in Stage IV.}

Given an initial value for the demand $d_{i}$ of each user $i$ Do the following procedure iteratively:

$$
\begin{aligned}
& \text { For each } 1 \leq i \leq N \text { : } \\
& \text { (1) Perform optimization for consumer } i \text { to find the } \\
& \text { optimal } d_{i} \text { value assuming that the power demands } \\
& \text { of the other users are given. We optimize Eqn. (8) } \\
& \text { for residential users or Eqn. (9) or PEVs. } \\
& \text { (2) Update the } d_{i} \text { value for consumer } i \text {. } \\
& \text { End }
\end{aligned}
$$

(1) Perform optimization for consumer $i$ to find the

Until the solution converges.

Since the factors $\beta$ and $\gamma$ are determined by the retailer, for different $\beta$ and $\gamma$ values, we can get different SPE solutions in Stage IV by applying Algorithm 1. We define two matrices Total_Demand and Total_Demand_Neg. Each entry Total_Demand $(\beta, \gamma)$ denotes the total demand $D$ of the users obtained from the SPE with given $\beta$ and $\gamma$ values provided by the retailer. Similarly Total_Demand_Neg $(\beta, \gamma)$ stores the corresponding $D_{\text {neg }}$ value. These matrices are used to reduce the computation complexity in the backward induction process.

\section{B. Optimization for The Retailer's Utility in Stage III}

The player in Stage III of the nested game is the retailer. In this stage, we are given the amount of electricity $D_{0}$ that the retailer acquires from energy sources. The optimization variables are the $\beta$ and $\gamma$ values. Because the $D_{0, \text { actual }}^{\text {NR }}$. price $^{\mathbf{N R}}$ and $D_{0, b u y}^{\mathbf{R}} \cdot$ price $^{\mathbf{R}}$ values in the original objective function (11) of the retailer are given in this stage, the retailer maximizes the following objective function:

$$
\left(D-D_{n e g}\right) \cdot p_{c}(D)+D_{n e g} \cdot p_{d}(D)-\theta \cdot\left(D-D_{0}\right)^{2}
$$

Please note that with given $\beta$ and $\gamma$ values, the corresponding $D$ and $D_{\text {neg }}$ values obtained from Stage IV are stored in entries Total_Demand $(\beta, \gamma)$ and Total_Demand_Neg $(\beta, \gamma)$, respectively. The retailer employs the ternary search method, which is an extension of the well-known binary search method, in order to find the optimal $\beta$ and $\gamma$ values assuming that objective function (12) is a quasi-concave function of $\beta$ and $\gamma$. In this stage, we define the matrix Obj_StepIII, where each entry Obj_StepIII $\left(D_{0}\right)$ denotes the value of objective function (12) with given $D_{0}$.

\section{Optimization for The Retailer's Utility in Stage II}

In this stage, the player is also the retailer. We are given the actual renewable energy $D_{0, \text { actual }}^{\mathbf{R}}$ obtained from the renewable power sources. The optimization variable is the amount of energy $D_{0, a c t u a l}^{\mathbf{N R}}$ for the retailer to purchase from the traditional power plants. Because the $D_{0, b u y}^{\mathbf{R}} \cdot$ price $^{\mathbf{R}}$ value in the original objective function (11) of the retailer is given in this stage, the retailer maximizes the following objective function in Stage II:

$$
\begin{gathered}
\left(D-D_{\text {neg }}\right) \cdot p_{c}(D)+D_{\text {neg }} \cdot p_{d}(D)-\theta \cdot\left(D-D_{0}\right)^{2} \\
-D_{0, \text { actual }}^{\mathrm{NR}} \cdot \text { price } \mathrm{NR}^{\mathrm{NR}}
\end{gathered}
$$

where $D_{0}=D_{0, \text { actual }}^{\mathbf{R}}+D_{0, \text { actual }}^{\mathbf{N R}}$. In this stage, we perform a simple search algorithm on $D_{0, \text { actual }}^{\mathrm{NR}}$ to find the optimal solution that maximizes objective function (13) based on $D_{0, \text { actual }}^{\mathbf{R}}$. Please note that the optimal value of $\left(D-D_{n e g}\right)$. $p_{c}(D)+D_{n e g} \cdot p_{d}(D)-\theta \cdot\left(D-D_{0}\right)^{2}$ (i.e., the first line of Eqn. (13)) is stored in matrix entry Obj_StepIII $\left(D_{0}\right)$ with given $D_{0}$ value.

In this stage, we define the matrix Obj_StepII after the optimization procedure. Each entry Obj_StepII $\left(D_{0, \text { actual }}^{\mathbf{R}}\right)$ stores the value of objective function (13) with given $D_{0, \text { actual }}^{\mathbf{R}}$.

\section{Optimization for The Retailer's Utility in Stage I}

In the first stage, the retailer determines the amount of electricity energy $D_{0, b u y}^{\mathbf{R}}$ to purchase from the renewable power sources. The objective function for the retailer is Eqn. (11). Similarly, we perform a simple search algorithm on $D_{0, \text { buy }}^{\mathbf{R}}$ to find the optimal solution, based on the matrix Obj_StepII and the realization factor $P_{\mathbf{R}}$ value. This optimization step is very critical for the retailer to improve its payoff. If the retailer purchases too much electricity from renewable power sources, the quality of steady power supply cannot be guaranteed due to the reliability issues. In contrast, acquiring electricity from conventional power plant only is not a cost-effective strategy because price $\mathbf{N R}^{\mathbf{R}}>$ price $^{\mathbf{R}}$.

\section{SimULATION RESUlTS}

In this section, we present the experimental results on the proposed nested-game based optimization framework. We have implemented an interactive simulation system of smart grid, including electricity retailer, PEVs and other electricity consumers. The effectiveness of the proposed optimization framework is demonstrated through comparison results.

In our simulation system we consider a user group of 20 consumers. Among all the consumers, 10 of them are residential users and the rest are PEVs. As is shown in residential user's utility function (8), $a_{i}$ is set to 2.5 for all the residential users and $d_{i}$ is set to a randomized value between 175 and 225. For PEV's utility function (9), $\varepsilon_{i}$ is set to a randomized value between 10 and 600 and $S_{\text {ini,i }}$ is uniformly distributed between 35 and 200 since each PEV has different storage of charge (SOC). We change the factor $\beta$ and $\gamma$ in realtime pricing Eqn. (6) and (7) between 0.05 and 0.3 , and $D_{0}$ from 50 to 180 to get the corresponding $D$ through algorithm 1 under different circumstances. And the base price $p_{B}$ is set to 8. In Stage I and II, price $\mathbf{N R}^{\mathbf{R}}$ is set to 15 and price $\mathbf{R}^{\mathbf{R}}$ is set to 4 and $\theta$ equals to 2 . 


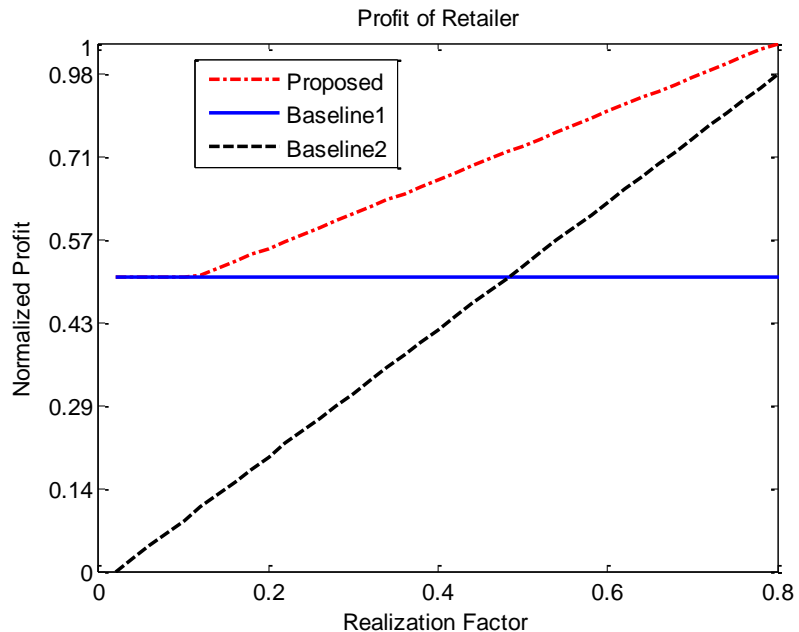

Fig. 2. Experiment results of retailer's profit.

In Figure 2, we compare our optimization results with two baseline results. For better comparison, we use normalized profit instead of its absolute value and we take the experimental result of our proposed algorithm at $P_{\mathbf{R}}=0.8$ as the normalization constant. For baseline 1, we assume that retailer acquire all the electricity from traditional power source. For baseline 2, we assume that retailer only buy electricity from renewable power sources. As we can see from the figure, when realization factor $P_{\mathbf{R}}$ becomes higher, retailer will get a better payoff if it acquires more electricity from renewable sources which is in low in unit price. Therefore, experiment results is a lot better than baseline1 when $P_{\mathbf{R}}>0.3$. Compared with baseline 2, the retailer's payoff will be less if there is too much electricity turn out to be not realizable. Obviously, when $P_{\mathbf{R}}<0.3$, our experimental result gets a higher payoff than that of baseline 2. Because the baseline 2 has reliability issues when the renewable energy becomes less reliable.

\section{CONCLUSION}

In this paper, we consider the interaction among energy sources, electricity retailer, and various consumers. The retailer procures electricity from both traditional and renewable energy sources, and sells it to its consumers using real time pricing policy. The consumers include residential users that can only consume power, and PEVs that can either consume power or supply power stored in its battery to the grid. We propose a novel four-stage nested game-based framework to model these interactions. The objective of the retailer is to maximize its overall profit as well as perform frequency regulation, whereas the goal of each consumer is to maximize a predefined utility function. In the game theoretic framework, the retailer should decide the amounts of electricity purchased from the renewable and traditional energy sources, respectively, and the real-time pricing scheme for its consumers. The consumers will react to the pricing mechanism and maximize their utility functions by adjusting the electricity demand. We derive the optimal solution of the nested game through finding the SPE of all the consumers and optimizing the retailer's action using the backward induction method.

\section{REFERENCES}

[1] M. Hashmi, S. Hannien, and K. Maki, "Survey of Smart Grid Concepts, Architectures, and Technological Demonstrations Worldwide," IEEE PES Conference on Innovative Smart Grid Technologies (ISGT Latin America), 2011.

[2] X. Fang, S. Misra and G. Xue, "Smart Grid - The New and Improved Power Grid: A Survey," IEEE Communications Surveys \& Tutorials, 2012.

[3] P. Samadi, R. Schober and V. Wong, "Optimal Energy Consumption Scheduling Using Mechanism Design for the Future Smart Grid," IEEE SmartGridComm, 2011.

[4] M. Shinwari, A. Youssef and W. Hamouda, "A Water-Filling Based Scheduling Algorithm for the Smart Grid," IEEE Transactions on Smart Grid, 2012.

[5] T. Cui, Y. Wang, S. Yue, S. Nazarian, and M. Pedram, "An incentivebased price determination algorithm for utility companies in smart grid under game theoretic models," in IEEE PES Innovative Smart Grid Technologies Conference (ISGT), 2013.

[6] C. Wu, H. Mohsenian-Rad, J. Huang, "Vehicle-to-Aggregator Interaction Game," IEEE Transactions on Smart Grid, vol. 3, No. 1, March 2012.

[7] W. Shi and V. Wong, "Real-Time Vehicle-to-Grid Control Algorithm under Price Uncertainty," IEEE SmartGridComm, 2011.

[8] S. Han, S. H. Han and K. Sezaki, "Design of An Optimal Aggregator for Vehicle-to-Grid Regulation Service," IEEE PES Innovative Smart Grid Technologies (ISGT), 2010.

[9] W. Saad, Z. Han and T. Basar, "Game-Theoretic Methods for the Smart Grid: An Overview of Microgrid Systems, Demand-Side Management, and Smart Grid Communications," IEEE Signal Processing Magazine, vol. 29, no. 5, pp. 86-105, Sept. 2012.

[10] P. Yang, G. Tang and A. Nehorai, "A Game-Theoretic Approach for Optimal Time-of-Use Electricity Pricing," IEEE Transactions on Power Systems, 2012.

[11] C. Wu, H. Mohsenian-Rad and J. Huang, "Wind Power Integration via Aggregator-Consumer Coordination: A Game Theoretic Approach," IEEE PES Innovative Smart Grid Technologies Conference (ISGT), Jan. 2012.

[12] S. Bu, F. R. Yu and P. X. Liu, "A Game-Theoretical Decision-Making Scheme for Electricity Retailers in the Smart Grid with Demand-Side Management," IEEE SmartGridComm, 2011.

[13] K. Leyton-Brown and Y. Shoham, Essentials of Game Theory: A Concise, Multidisciplinary Introduction, Morgan \& Claypool Publishers, 2008.

[14] J. B. Rosen, "Existence and uniqueness of equilibrium points for concave $n$-person games," Econometrica, vol. 33, pp. 347-351, 1965.

[15] S. Boyd and L. Vandenberghe, Convex Optimization, Cambridge University Press, 2004. 